Check for updates

Cite this: Phys. Chem. Chem. Phys., 2017, 19, 16875

Received 7th April 2017, Accepted 9th June 2017 DOI: $10.1039 / c 7 c p 02251 d$

rsc.li/pccp

\title{
Surface-specific vibrational spectroscopy of the water/silica interface: screening and interference $\uparrow$
}

\author{
Jan Schaefer, (D) Grazia Gonella, (D) Mischa Bonn (D) and Ellen H. G. Backus (DD *
}

\begin{abstract}
Surface-specific vibrational sum-frequency generation spectroscopy (V-SFG) is frequently used to obtain information about the molecular structure at charged interfaces. Here, we provide experimental evidence that not only screening of surface charges but also interference limits the extent to which V-SFG probes interfacial water at sub-mM salt concentrations. As a consequence, $\mathrm{V}$-SFG yields information about the $\sim$ single monolayer interfacial region not only at very high ionic strength, where the surface charge is effectively screened, but also for pure water due to the particularly large screening length at this low ionic strength. At these low ionic strengths, the large screening lengths cause destructive interference between contributions in the surface region. A recently proposed theoretical framework near-quantitatively describes our experimental findings by considering only interference and screening. However, a comparison between $\mathrm{NaCl}$ and $\mathrm{LiCl}$ reveals ion specific effects in the screening efficiency of different electrolytes. Independent of electrolyte, the hydrogen bonding strength of water right at the interface is enhanced at high electrolyte concentrations.
\end{abstract}

\section{Introduction}

In contact with water, mineral surfaces may accumulate an excess of positive or negative charges, which in turn affects the arrangement of interfacial water molecules. Through interaction with the associated DC electric field, interfacial water layers form the so-called electric double layer (EDL) which consists of the near-surface, highly ordered Stern layer, and the more diffuse outer water layers. It is clear that interfacial chemistry, relevant for many geological and industrial processes, is affected by the reaction of dipolar interfacial molecules, in particular water, to the surface field. The distance-dependent potential associated to such an electric field is typically described by the Gouy-Chapman-Stern model, assuming an exponential decay of the potential away from the surface through the diffuse layer, while the Stern layer is characterized by a linear drop in analogy to a dielectric capacitor.

Associated parameters such as surface potential, surface charge density and Debye screening length are challenging to access experimentally. Besides potentiometric titration, ${ }^{1,2}$ Atomic Force Microscopy ${ }^{3}$ and X-ray Photoelectron Spectroscopy, ${ }^{4,5}$ also non-linear spectroscopy techniques have been demonstrated to be a useful tool for studying these kind of interfaces. ${ }^{6-18}$ As such, we employed Vibrational Sum Frequency Generation (V-SFG) spectroscopy for interrogating those interfacial properties, since

Max Planck Institute for Polymer Research, Ackermannweg 10, 55128, Mainz, Germany.E-mail: backus@mpip-mainz.mpg.de

$\dagger$ Electronic supplementary information (ESI) available. See DOI: 10.1039/c7cp02251d it allows for probing vibrational states of interfacial molecules with sub-micrometer sensitivity and selectivity towards the symmetry-broken interfacial layers (given by the additional symmetry selection rule underlying V-SFG processes). Using this technique, we study how the presence of ions changes the composition of the EDL for water at a mineral surface. Specifically, we aim to understand to which depth water interacts with the surface electric field and if the intermolecular structure of interfacial water is affected differently by the presence of ions than that of bulk water. For this purpose, we investigated the silica/water interface with varying electrolyte content. Around neutral $\mathrm{pH}$, the silica surface is covered by silanol groups which are mostly deprotonated, generating a negatively charged surface as the point of zero charge (pzc) is $\mathrm{pH} 2 .^{1,14}$

V-SFG spectra of neutral interfaces are commonly discussed by invoking the second-order non-linear susceptibility $\chi^{(2)}$ of involved media. Based on symmetry arguments, $\chi^{(2)}$ is zero for centro-symmetric materials. This makes V-SFG a sensitive probe for the first few molecular layers of e.g. water at interfaces, as only at the interface centro-symmetry is broken. Moreover, $\chi^{(2)}$ is proportional to molecular order and molecular number density. However, as discussed previously, ${ }^{6,10,19-21}$ this description does not suffice in presence of charged interfaces since surface charges may induce an extra DC electric field, $E_{\mathrm{DC}}$, which, in addition to the IR- and VIS- fields, $E_{\mathrm{IR}}$ and $E_{\mathrm{VIS}}$, also acts on nonlinear active molecules in the proximity of the surface. This gives rise to an additional third order $\left(\chi^{(3)}\right)$ contribution to the V-SFG response which depends on the penetration depth of the DC field and may even exceed the $\chi^{(2)}$-response. As a consequence, 
the generated V-SFG signal contains a $\chi^{(2)}$ contribution from directly bonded interfacial molecules and a $\chi^{(3)}$ contribution from up to $\sim 1 \mu \mathrm{m}$ away from the surface for low ion concentrations. ${ }^{15}$ In contrast to $\chi^{(2)}$, not only oriented but also isotropic water may contribute to $\chi^{(3)}$ signal, i.e. $\chi_{\text {aniso }}^{(3)}$ and $\chi_{\text {iso }}^{(3)}$, where the anisotropic term reflects the DC-field induced orientation of near-surface water, and the isotropic term the contribution from bulk-like, randomly oriented water. As such, the V-SFG intensity of charged interfaces does not purely reflect the degree of interfacial order but is a convolution of field induced reorientation and polarization: ${ }^{6,10,22}$

$$
\begin{gathered}
I(\mathrm{SFG}) \propto\left|E_{\mathrm{VIS}} E_{\mathrm{IR}} \chi^{(2)}+\int_{0}^{+\infty} E_{\mathrm{VIS}} E_{\mathrm{IR}} E_{\mathrm{DC}}(z) \chi^{(3)} \mathrm{d} z\right|^{2} \\
\chi^{(3)}=\chi_{\text {aniso }}^{(3)}+\chi_{\text {iso }}^{(3)}
\end{gathered}
$$

It is clear from eqn (1) that the V-SFG intensity of such systems depends strongly on the number of molecules interacting with the DC electric field. That number is determined by the strength and decay length of that field, which, in turn are defined by the charge density at the surface and the Debye screening length of the solution. With increasing bulk ion concentration, the surface charges are increasingly shielded, resulting in reduced penetration depths of the DC field. Conversely, with increasing Debye length (i.e. decreasing bulk ion concentration) an increasing number of molecules contribute, resulting in a higher V-SFG signal. For a given surface charge, the probing depth can thus be tuned by varying the ionic content in the solution.

As the V-SFG signal of charged interfaces is thus composed of a complex interplay between the bonded interfacial layer $\chi^{(2)}$ and the diffuse layer $\chi^{(3)}$ response, it is a priori not clear how these contributions are convoluted in the V-SFG spectrum. For the silica/water interface, the correlation between V-SFG intensity and bulk ion concentration has been studied experimentally for aqueous sodium chloride solutions between $10^{-4} \mathrm{M}$ and $4 \mathrm{M}^{10}$ The decrease of the V-SFG intensity with increasing electrolyte concentration has mainly been attributed to Debye screening due to the presence of electrolyte. However, a second electrolyte concentration-dependent effect has been proposed, based on theoretical considerations and direct observation by second harmonic scattering. ${ }^{15,23}$ At very low electrolyte concentrations, the probing depth can be limited by phase matching of the V-SFG probe. Hence, as the penetration depth of the DC field exceeds the coherence length, the signal intensity is expected to be increasingly depleted due to destructive interference between contributions from sufficiently spatially separated non-linear active molecules. ${ }^{15}$ For particularly large penetration depths, the third order contribution vanishes completely and only the field-independent V-SFG response of the bonded interfacial layer $\left(\chi^{(2)}\right)$ survives. $^{15}$ The V-SFG intensity may thus not necessarily increase with number of molecules affected by the DC field as has been generally assumed, ${ }^{6,7,10,20,24,25}$ but decreases if the DC field reaches the magnitude of the coherence length of the probed non-linear process. ${ }^{15,17}$
Here, we provide new insights into the sparsely studied regime of low ionic strength $\left([\mathrm{NaCl}]<5 \times 10^{-4} \mathrm{M}\right)$ for which we observe an inverted dependence between ionic strength and the V-SFG signal intensity in accordance with theoretical predictions. $^{15}$ We demonstrate experimentally that V-SFG spectroscopy provides interfacial layer sensitivity to the silica/ water interface both for pure water and for highly concentrated $(>1 \mathrm{M}) \mathrm{NaCl}$ solutions. Furthermore, we show that the overall concentration dependence of the V-SFG intensity is similar for monovalent cations of different radii, however they seem to differ in their screening efficiency. Moreover, we observe significant redshifts of the $\mathrm{O}-\mathrm{H}$ stretch signal for high electrolyte concentration, which can only be rationalized by invoking surface-specific effects. Apparently, salt has a different impact on water molecules close to the surface than on water molecules in the bulk.

\section{Results and discussion}

The top panel in Fig. 1 depicts V-SFG spectra of the silica/water interface within the $\mathrm{C}-\mathrm{H}-/ \mathrm{O}-\mathrm{H}$-stretch frequency window for three different $\mathrm{NaCl}$ concentrations. The spectra show the typical double peak O-H feature between $\sim 2900$ and $3500 \mathrm{~cm}^{-1}$, as discussed in earlier studies of water in contact with silica surfaces. ${ }^{7-10}$ Due to the absence of any $\mathrm{C}-\mathrm{H}$ vibrational signatures, there is no indication of surface contamination with organic impurities. However, we caution that the preparation of consistent silica surfaces itself is challenging since charged surfaces might adsorb unwanted moieties. Compared to pure water and the $1 \mathrm{M} \mathrm{NaCl}$ solution, which show similar intensity, the signal from the $5 \times 10^{-4}$ molar solution is significantly enhanced. The presented spectra in Fig. 1 are raw data, not normalized to the spectrum of the IR pulse as we are only interested in relative changes of the $\mathrm{O}-\mathrm{H}-\mathrm{band}$. The normalized spectra can be found in the ESI $\dagger$ (Fig. S1). For a dilution series of 20 different $\mathrm{NaCl}$ concentrations, we integrate the corresponding spectra between 2800 and $3600 \mathrm{~cm}^{-1}$, and the resulting integrated signals are plotted as a function of the ion concentration in the bottom panel of Fig. 1 .

As illustrated in Fig. 1, the V-SFG intensity of the $\mathrm{O}-\mathrm{H}$ stretching band dramatically depends on the bulk ion concentration. Overall, we observe an order of magnitude variation in the V-SFG signal upon the addition of $\mathrm{NaCl}$ to pure water. At both low and high concentration, the signal is small, while a maximum in the V-SFG intensity appears at $\sim 5 \times 10^{-4} \mathrm{M} \mathrm{NaCl}$. SFG experiments with SPS polarization reveal a very similar trend, so that the dependence of the SFG intensity on electrolyte concentration cannot be accounted for by concentration-dependent orientation of interfacial water molecules. This decrease of the V-SFG intensity for $\mathrm{NaCl}$ concentrations exceeding $5 \times 10^{-4} \mathrm{M}$ has been observed by Jena et al. ${ }^{10}$ In that study, four different regimes of ion concentration have been distinguished (A-D). In a similar fashion, we will now discuss our results and provide new insight into the concentration regime below $5 \times 10^{-4} \mathrm{M}$ (A). For the high concentration regimes (B-D), our results agree with the above mentioned observations ${ }^{10}$ which we only summarize here. 

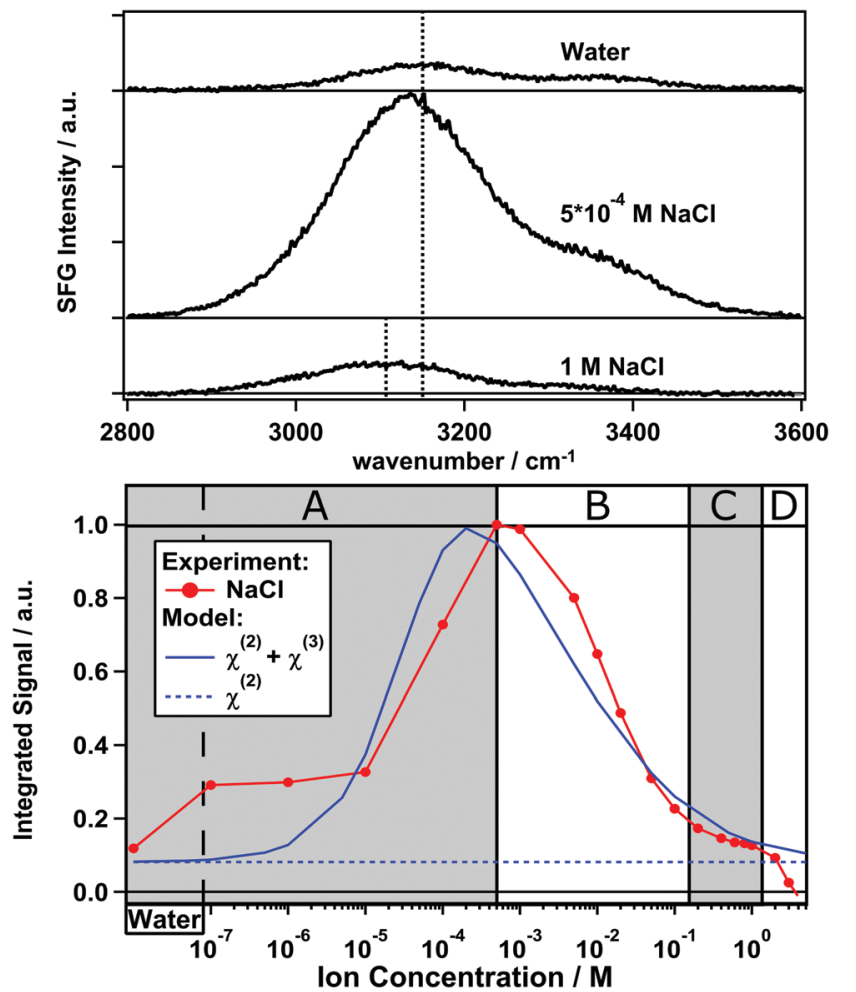

Fig. 1 Top panel: Non-normalized $\mathrm{O}-\mathrm{H}$-stretch $\mathrm{V}$-SFG signal from the silica/water interface for pure water, $5 \times 10^{-4} \mathrm{M}$ and $1 \mathrm{M} \mathrm{NaCl}$ solutions. The black dashed lines mark the frequency at peak maximum for pure water and the $1 \mathrm{M} \mathrm{NaCl}$ solution. Bottom panel: Integrated V-SFG signal (red) as a function of $\mathrm{NaCl}$ concentration and the predicted intensity (blue solid) by invoking modulation by charge screening and interference (eqn (4)). ${ }^{15}$ The blue dashed line represents the considered field-independent, true second order contribution to the V-SFG response.

Regime B: for $[\mathrm{NaCl}]>5 \times 10^{-4} \mathrm{M}$, the V-SFG signal decreases with increasing ion concentration due to increased screening.

Regime C: for high ionic strengths of around $1 \mathrm{M}$, the intensity is almost constant which indicates that the number of probed molecules and their orientation do not change substantially within this regime. It has been argued before that the signal in this region corresponds mostly to the intrinsic $\chi^{(2)}$ response, providing insight into the Stern layer of the silica/water interface. ${ }^{10}$

Regime D: for concentrations higher than $2 \mathrm{M}$, the intensity drops more rapidly which suggests either randomization of Stern layer water molecules or replacement by ions directly adsorbing on the silica surface.

Regime A: in contrast to Jena et al., ${ }^{10}$ we find that in this regime where $[\mathrm{NaCl}]<5 \times 10^{-4} \mathrm{M}$, the V-SFG intensity decreases with further decreasing salt concentration. For neat water, we observe a very low V-SFG signal. To avoid contamination due to dissolution of silica which is known to affect the V-SFG signal, ${ }^{24}$ we flushed the cell with sample solution before starting each measurement. This finding can be rationalized, according to the model in ref. 15, by considering the Debye screening length of pure water to be larger than the coherence length of the V-SFG process in the given experimental geometry
( $\sim 40 \mathrm{~nm})$ which gives rise to destructive interference and cancellation of the $\chi^{(3)}$ contribution. As the penetration depth of the DC field decreases upon adding salt, phase matching is increasingly fulfilled by approaching the coherence length, which results in a higher V-SFG intensity.

For understanding our data on a quantitative level, we employed the model for predicting the non-linear response as proposed in ref. 15 which uses a Gouy-Chapman description of the EDL and phase matching condition of the non-linear process as given by:

$$
I(\mathrm{SFG}) \propto\left|E_{\mathrm{VIS}} E_{\mathrm{IR}}\left(\chi^{(2)}+\chi^{(3)} \int_{0}^{+\infty} E_{\mathrm{DC}}(z) e^{i \Delta k_{z} z} \mathrm{~d} z\right)\right|^{2}
$$

where $E_{\mathrm{DC}}$ is the surface DC field, and $\Delta k_{z}$ represents the phase mismatch ${ }^{20}$ accumulated for different points along the coordinate perpendicular to the silica/water interface $z$. By performing the integration with $E_{\mathrm{DC}}(z)=-\frac{\mathrm{d} \phi(z)}{\mathrm{d} z}$ and by considering an exponentially decaying potential $\phi(z)=\phi_{0}(c) \times \exp (-\kappa(c) z)$ along the $z$-direction (perpendicular to the silica/water interface), the V-SFG intensity dependence on ionic strength can be formulated as follows:

$$
I(\mathrm{SFG}) \propto\left|E_{\mathrm{VIS}} E_{\mathrm{IR}}\left(\chi^{(2)}+\chi^{(3)} \phi_{0}(c) \frac{\kappa(c)}{\kappa(c)-i \Delta k_{z}}\right)\right|^{2}
$$

Besides the intensity of the involved optical beams and the non-linear susceptibilities of water, the resulting V-SFG intensity is determined by the ionic strength-dependent Debye length, $\kappa(c)$, and surface potential, $\phi_{0}(c)$, (as described by the Grahame equation) as well as the phase mismatch along the probing depth, $\Delta k_{z}$, whose explicit definitions are given in the $\mathrm{ESI} \dagger$ (eqn (S1)-(S3)). As can be seen in eqn (S1)-(S3) (ESI $\dagger$ ), the surface potential is also directly dependent on the surface charge density, $\sigma_{0}$, and the relative permittivity of the aqueous medium, $\varepsilon_{\mathrm{r}}$. For the V-SFG intensity vs. concentration curve generated in Fig. 1, we have used $\sigma_{0}=-0.05 \mathrm{C} \mathrm{m}^{-2}$ and $\varepsilon_{\mathrm{r}}=78$ as suggested in ref. 15. Through comparison with our experimental data, we infer a $\chi^{(3)} / \chi^{(2)}$ ratio of $-17.5 \mathrm{~V}^{-1}$. Within the range of reported surface charge densities for fused silica around neutral $\mathrm{pH},-0.06 \mathrm{C} \mathrm{m}^{-2}<\sigma_{0}<-0.02 \mathrm{C} \mathrm{m}^{-2,2,4,14,26,27}$ we find that comparable intensity curves can be obtained for $-22.25 \mathrm{~V}^{-1}<\chi^{(3)} / \chi^{(2)}<-16.50 \mathrm{~V}^{-1}$ as reported in the ESI $\dagger$ (Fig. S2). These values for the $\chi^{(3)} / \chi^{(2)}$ ratio seem reasonable compared with previous studies of water at charged surfaces. ${ }^{20,23}$ Please note that for simplicity we assume that the charge density is constant for all salt concentrations. Small variation with concentration cannot be excluded as the surface charge density of silica $\left(-0.06 \mathrm{C} \mathrm{m}^{-2}<\sigma_{0}<-0.02 \mathrm{C} \mathrm{m}^{-2}\right)^{2,4,14,26,27}$ is typically around the critical surface charge density $\left(\sigma_{0, \text { crit }}\right)$ above which counterion condensation occurs. $^{28}$

We observe that the shape of the V-SFG intensity $v s$. ion concentration curve generated by the model agrees remarkably well with our experimental data. As predicted, pure water shows similar intensity as $\mathrm{NaCl}$ solutions within regime $\mathrm{C}$ (Fig. 1) while the intensity is enhanced for an intermediate concentration 
regime with its maximum around $[\mathrm{NaCl}]=5 \times 10^{-4} \mathrm{M}$. Based on the employed theoretical framework, ${ }^{15}$ this indicates that the $\chi^{(2)}$-contribution (blue dashed line in Fig. 1) dominates the pure water response and interfacial layer sensitivity is obtained not only at short but also at very large screening lengths. In contrast, the probing depth amounts to a few tens of nanometers for the maximum at $5 \times 10^{-4} \mathrm{M} \mathrm{NaCl}$. In its current form, the model seems to overestimate the screening contribution of $\mathrm{NaCl}$, reducing the $\mathrm{V}-\mathrm{SFG}$ intensity in the $\mathrm{B}$ and $\mathrm{C}$ regime and shifting the maximum to lower concentration than what is observed in the experiment.

While eqn (4) discriminates between ions species with different valence, the effect of different ionic radii is ignored. However, ion size not only determines the Stern-layer thickness, ${ }^{5}$ but might also be relevant for the screening ability since the ion mobility changes with the hydrated radius. For a negatively charged surface, the properties of the cations are especially important. $^{13,29}$ Therefore, we compare two not-too-different alkali ions, $\mathrm{Na}^{+}$and $\mathrm{Li}^{+}$, where however $\mathrm{Li}^{+}$shows a lower ion mobility due to a smaller ionic but larger hydrated radius. In the same fashion as presented in Fig. 1, we will now discuss the results of two measurement series for both $\mathrm{NaCl}$ and $\mathrm{LiCl}$ solutions which are shown in Fig. 2. Again, the integrated signal is plotted as a function of ion concentration and the curves are each normalized to their maximum.

Overall, the intensity curve shape for LiCl is very similar to that already discussed for $\mathrm{NaCl}$ solutions in Fig. 1. However, the curves for $\mathrm{LiCl}$ are shifted to slightly higher concentrations than those for $\mathrm{NaCl}$. Again, in regime $\mathrm{B}-\mathrm{D}$, the intensity decreases with increasing ion concentration while regime $\mathrm{A}$ shows inverted concentration dependence. By comparing the two measurement series for $\mathrm{NaCl}$, we find a slightly varying ratio between the maximum intensity and the plateau in regime A and $\mathrm{C}$ indicating a small variability of the surface charge on silica, which can likely be traced to slight, unintentional differences in the preparation of the surface.

Besides the shift of the curve toward higher concentration for $\mathrm{LiCl}$ compared to $\mathrm{NaCl}$, we observe a steeper intensity increase within regime $\mathrm{B}-\mathrm{C}$ which suggests that for generating the same screening length, a higher $\mathrm{LiCl}$ concentration is required than for $\mathrm{NaCl}$. In other words, $\mathrm{Na}^{+}$ions seem to shield surface charges more effectively than $\mathrm{Li}^{+}$ions but both are still less efficient than predicted by eqn (4) as shown in Fig. 1. Additionally, the intensity plateau within regime $\mathrm{C}$ is less pronounced for $\mathrm{LiCl}$ than for $\mathrm{NaCl}$. This indicates that the bonded interfacial layer of water gets perturbed and/or replaced more easily by $\mathrm{Li}^{+}$than $\mathrm{Na}^{+}$ions. In summary, we observe an impact of the ionic radius on the V-SFG intensity curves which seems to be a consequence of a higher screening efficiency for larger cations with equal valence. One explanation may be that alkali ions with larger ionic radii are less hydrated providing higher ion mobility in aqueous media. Consequently, an external field may attract the highly mobile $\mathrm{Na}^{+}$ions more easily than $\mathrm{Li}^{+}$ ions. However, at short distance to the surface, $\mathrm{Na}^{+}$seems to show a lower surface propensity than $\mathrm{Li}^{+}$, as reflected by the $\mathrm{O}-\mathrm{H}$-stretch V-SFG intensity plateau at high $\mathrm{NaCl}$ concentration. Both findings

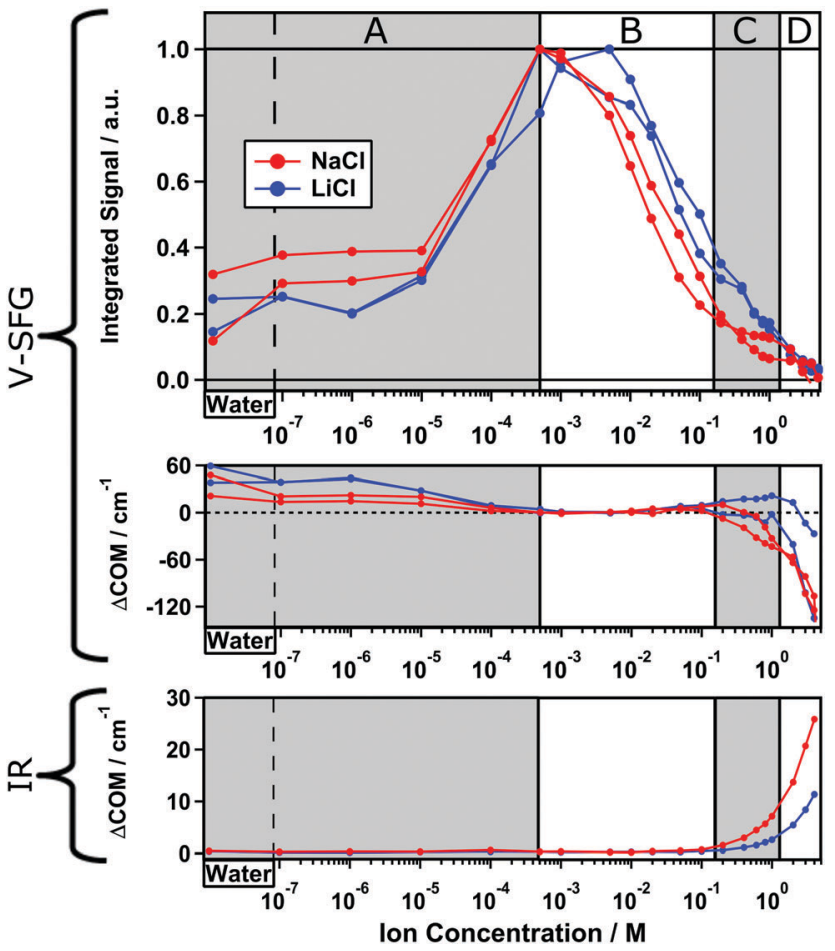

Fig. 2 Two experiments for two dilution series of $\mathrm{NaCl}$ (red) and $\mathrm{LiCl}$ (blue). Top panel: Integrated O-H-stretch V-SFG signal. Middle panel: Center of mass frequency shifts of the corresponding signals, each referenced to the maximum signal at $5 \times 10^{-4} \mathrm{M} \mathrm{NaCl}$. Bottom panel: Center of mass frequency shifts of the $\mathrm{O}-\mathrm{H}$-stretch IR signals.

are in line with recent atomic force microscopy studies and molecular dynamics simulations. ${ }^{3,30}$

Second harmonic generation (SHG) spectroscopy has been widely employed for studying the silica/water interface., 6,11-14,16,17 In contrast to V-SFG, SHG probes a non-linear signal that is not resonantly enhanced and therefore not sensitive to a particular molecule or moiety but may result from any molecular and atomic species present which is in particular relevant for high salt concentrations. Generally speaking, any added substance could cause an unintentional increase of the SHG response. Azam et al. have shown that, within regime C, opposed to our V-SFG results, the SHG intensity has inverted concentration dependence for $\mathrm{LiCl}$ as compared to $\mathrm{NaCl}$. This observation has been attributed to ion specific effects on the surface charge. ${ }^{13}$ Boamah et al. have demonstrated that for $\mathrm{NaCl}$, the SHG intensity does not drop to zero at high ionic strength and this has been rationalized by assuming either dramatic changes in the surface charge density of silica or in the relative permittivity of water in the diffuse layer. ${ }^{17}$ Our data do not show this effect. These comparisons seem to indicate that a direct probe of interfacial molecules or functional groups, as provided with V-SFG, is helpful for understanding the decaying surface potential at charged surfaces in contact with an aqueous solution.

As discussed above, V-SFG seems to provide interfacial layer sensitivity at the silica/water interface for pure water and $\sim 1 \mathrm{M}$ ion concentration. As V-SFG is a resonant probe of vibrational states, here the $\mathrm{O}-\mathrm{H}$ stretching of interfacial water, the V-SFG 
spectrum can provide insights into the interfacial interactions through comparison of band frequencies. From the upper panel of Fig. 1 it is evident that the $\mathrm{O}-\mathrm{H}$ stretching band is shifting upon changing the salt concentration. For a quantitative analysis, we calculated the spectral first moment ('center of mass', COM) for the corresponding spectra and compared changes relative to the largest signal $\left(5 \times 10^{-4}\right.$ and $5 \times 10^{-3} \mathrm{M}$ for $\mathrm{NaCl}$ and LiCl, respectively) as depicted in the middle panel of Fig. 2.

For all measurement series, we consistently observe an overall red-shifting $\mathrm{O}-\mathrm{H}$-stretch $\mathrm{V}$-SFG band upon increasing ion concentration - Fig. 2, middle panel. In contrast, corresponding bulk spectra show a blue-shifting $\mathrm{O}-\mathrm{H}$-stretch band as illustrated in the lower panel of Fig. 2 for IR measurements and reported in ref. 31 for Raman. Therefore, the ion-induced red-shift of the SFG band seems to be a purely surface-related effect. In between these two ionic concentrations at which we measure an SFG response originating purely from the outermost interfacial layer, i.e. pure water and $\sim 1 \mathrm{M}$ electrolyte, we observe a red-shift of about $60 \mathrm{~cm}^{-1}$ which suggests that interfacial water in presence of salt has substantially stronger hydrogen bonds.

\section{Conclusion}

To the best of our knowledge, this work is the first experimental evidence that interference limits the extent to which SFG probes the interface of water in contact with a charged surface such as silica. The maximum SFG signal is generated around millimolar ionic strength which for a 1:1 electrolyte corresponds to a Debye length of tens of nanometers. As a consequence of interference, not only short, but also large Debye lengths, as present for pure water, provide nearly monolayer insight into the interfacial region. The proposed model for the ionic-strength dependent non-linear response from a charged surface, using previously reported surface charge densities for silica and an exponentially decaying surface potential, can be used for describing our SFG data. However, the decay length of the surface potential seems to depend not only on the ion valence: In fact, our data suggest that ions with smaller hydration radii screen surface charges more efficiently, while the Stern layer water is perturbed more easily by ions with larger hydration radii. Irrespective of the ion identity, the $\mathrm{O}-\mathrm{H}$ stretch SFG band undergoes a redshift with increasing bulk ionic strength as opposed to a blueshift observed for bulk water which indicates that the intermolecular structure of interfacial water at the silica surface changes differently in presence of salt than what observed in bulk. We interpret the observed red-shift as a strengthening of the interfacial H-bonding structure.

\section{Experimental methods}

\section{Spectroscopy}

The SFG spectra presented in this work are recorded with an experimental setup that uses a Ti:sapphire regenerative amplifier (Solstice $^{\mathbb{R}}$ Ace $^{\mathrm{TM}}$, Spectra Physics) which generates $800 \mathrm{~nm}$ pulses with $\sim 40$ fs duration and $1 \mathrm{kHz}$ repetition rate. For producing broadband $\left(\sim 450 \mathrm{~cm}^{-1}\right)$ infrared pulses with $5 \mu \mathrm{J}$, a commercial optical parametric amplifier (TOPAS Prime, Spectra Physics) is used together with a non-collinear difference frequency generation (NDFG) scheme. By guiding the visible upconverting pulse through a Fabry-Perot etalon (SLS Optics Ltd), it is spectrally narrowed to a FWHM of $\sim 20 \mathrm{~cm}^{-1}$ with $\sim 20 \mu \mathrm{J}$. The SFG signal is spectrally resolved using a spectrograph (Acton Spectro Pro ${ }^{\circledR}$ SP-2300, Princeton Instruments) and detected with an electronmultiplied charge couple device (emCCD) camera (ProEM 1600, Roper Scientific). All spectra are recorded in ssp polarization combination (s-polarized SFG, s-polarized visible and p-polarized IR) and at incident angles $\theta_{\mathrm{Vis}} \approx 37^{\circ}$ and $\theta_{\mathrm{IR}} \approx 33^{\circ}$. Each spectrum results from 10 minute accumulation. For normalization, the frequency dependent IR field is measured on an equally prepared silica window, coated with $100 \mathrm{~nm}$ chromium-free gold.

The IR data presented in the lower panel of Fig. 2 result from ATR measurements accomplished with a Bruker Tensor II (Platinum ATR) and are corrected for the penetration depth in pure water by using the refractive index as reported in ref. 32 .

\section{Sample preparation}

Lithium chloride and sodium chloride, purchased from Sigma Aldrich ( $\geq 99.5 \%$, CAS 7647-14-5) and Roth ( $\geq 99.5 \%$, CAS 744741-8), respectively, are dissolved in demineralized $\mathrm{H}_{2} \mathrm{O}$, filtered with a Millipore unit (resistivity $=18 \mathrm{M} \Omega \mathrm{cm}$ ). For removing organic residues, sodium chloride is baked at $650{ }^{\circ} \mathrm{C}$ for 6 hours. Before each experiment, the (parallel faced) fused silica window (Korth Kristalle GmbH Infrasil ${ }^{\circledR} 302$, s/d: 60/40) is treated by UV-Ozone cleaning for $30 \mathrm{~min}$. All sample solutions are measured at least one hour after preparation to ensure $\mathrm{CO}_{2}$-equilibration. Before each measurement, the cell (as detailed in ref. 24$)$ is flushed with the sample solution $\left(V_{\text {total }}=100 \mathrm{~mL}\right)$ for 5 minutes $\left(500 \mathrm{~mL} \mathrm{~min}^{-1}\right.$ ).

\section{Acknowledgements}

We thank Dan Lis for initiating this project. This work was funded by an ERC Starting Grant (Grant No. 336679). Open Access funding provided by the Max Planck Society.

\section{References}

1 P. M. Dove and C. M. Craven, Surface Charge Density on Silica in Alkali and Alkaline Earth Chloride Electrolyte Solutions, Geochim. Cosmochim. Acta, 2005, 69, 4963-4970.

2 M. Karlsson, C. Craven, P. M. Dove and W. H. Casey, Surface Charge Concentrations on Silica in Different 1.0 M MetalChloride Background Electrolytes and Implications for Dissolution Rates, Aquat. Geochem., 2001, 7, 13-32.

3 J. Morag, M. Dishon and U. Sivan, The Governing Role of Surface Hydration in Ion Specific Adsorption to Silica: An AFM-Based Account of the Hofmeister Universality and Its Reversal, Langmuir, 2013, 29, 6317-6322. 
4 M. A. Brown, A. Goel and Z. Abbas, Effect of Electrolyte Concentration on the Stern Layer Thickness at a Charged Interface, Angew. Chem., Int. Ed., 2016, 55, 3790-3794.

5 M. A. Brown, Z. Abbas, A. Kleibert, R. G. Green, A. Goel, S. May and T. M. Squires, Determination of Surface Potential and Electrical Double-Layer Structure at the Aqueous ElectrolyteNanoparticle Interface, Phys. Rev. X, 2016, 6, 011007.

6 S. Ong, X. Zhao and K. B. Eisenthal, Polarization of Water Molecules at a Charged Interface: Second Harmonic Studies of the Silica/Water Interface, Chem. Phys. Lett., 1992, 191, 327-335.

7 V. Ostroverkhov, G. A. Waychunas and Y. R. Shen, Vibrational Spectra of Water at Water/ $\alpha$-Quartz (0001) Interface, Chem. Phys. Lett., 2004, 386, 144-148.

8 V. Ostroverkhov, G. A. Waychunas and Y. R. Shen, New Information on Water Interfacial Structure Revealed by Phase-Sensitive Surface Spectroscopy, Phys. Rev. Lett., 2005, 94, 046102.

9 K. C. Jena and D. K. Hore, Variation of Ionic Strength Reveals the Interfacial Water Structure at a Charged Mineral Surface, J. Phys. Chem. C, 2009, 113, 15364-15372.

10 K. C. Jena, P. A. Covert and D. K. Hore, The Effect of Salt on the Water Structure at a Charged Solid Surface: Differentiating Second- and Third-Order Nonlinear Contributions, J. Phys. Chem. Lett., 2011, 2, 1056-1061.

11 M. S. Azam, C. N. Weeraman and J. M. Gibbs-Davis, Specific Cation Effects on the Bimodal Acid-Base Behavior of the Silica/Water Interface, J. Phys. Chem. Lett., 2012, 3, 1269-1274.

12 M. S. Azam, C. N. Weeraman and J. M. Gibbs-Davis, HalideInduced Cooperative Acid-Base Behavior at a Negatively Charged Interface, J. Phys. Chem. C, 2013, 117, 8840-8850.

13 M. S. Azam, A. Darlington and J. M. Gibbs-Davis, The Influence of Concentration on Specific Ion Effects at the Silica/ Water Interface, J. Phys.: Condens. Matter, 2014, 26, 244107.

14 A. M. Darlington and J. M. Gibbs-Davis, Bimodal or Trimodal? The Influence of Starting pH on Site Identity and Distribution at the Low Salt Aqueous/Silica Interface, J. Phys. Chem. C, 2015, 119, 16560-16567.

15 G. Gonella, C. Lütgebaucks, A. G. de Beer and S. Roke, Second Harmonic and Sum-Frequency Generation from Aqueous Interfaces Is Modulated by Interference, J. Phys. Chem. C, 2016, 120, 9165-9173.

16 P. E. Ohno, S. A. Saslow, H.-F. Wang, F. M. Geiger and K. B. Eisenthal, Phase-Referenced Nonlinear Spectroscopy of the $\alpha$-Quartz/Water Interface, Nat. Commun., 2016, 7, 13587-13591.

17 M. D. Boamah, P. E. Ohno, F. M. Geiger and K. B. Eisenthal, Relative Permittivity in the Electrical Double Layer from Nonlinear Optics, 2017, arXiv:1702.02496v1.

18 L. Dalstein, E. Potapova and E. Tyrode, The Elusive Silica/ Water Interface: Isolated Silanols under Water as Revealed by Vibrational Sum Frequency Spectroscopy, Phys. Chem. Chem. Phys., 2017, 19, 10343-10349.
19 D. E. Gragson and G. L. Richmond, Investigations of the Structure and Hydrogen Bonding of Water Molecules at Liquid Surfaces by Vibrational Sum Frequency Spectroscopy, J. Phys. Chem. B, 1998, 102, 3847-3861.

20 Y.-C. Wen, S. Zha, X. Liu, S. Yang, P. Guo, G. Shi, H. Fang, Y. R. Shen and C. Tian, Unveiling Microscopic Structures of Charged Water Interfaces by Surface-Specific Vibrational Spectroscopy, Phys. Rev. Lett., 2016, 116, 016101.

21 A. Eftekhari-Bafrooei and E. Borguet, Effect of Surface Charge on the Vibrational Dynamics of Interfacial Water, J. Am. Chem. Soc., 2009, 131, 12034-12035.

22 B. Levine and C. G. Bethea, Second and Third Order Hyperpolarizabilities of Organic Molecules, J. Chem. Phys., 1975, 63, 2666-2682.

23 C. Lütgebaucks, G. Gonella and S. Roke, Optical Label-Free and Model-Free Probe of the Surface Potential of Nanoscale and Microscopic Objects in Aqueous Solution, Phys. Rev. B: Condens. Matter Mater. Phys., 2016, 94, 195410.

24 D. Lis, E. H. G. Backus, J. Hunger, S. H. Parekh and M. Bonn, Liquid Flow Along a Solid Surface Reversibly Alters Interfacial Chemistry, Science, 2014, 344, 1138-1142.

25 P. A. Covert, K. C. Jena and D. K. Hore, Throwing Salt into the Mix: Altering Interfacial Water Structure by Electrolyte Addition, J. Phys. Chem. Lett., 2013, 5, 143-148.

26 S. H. Behrens and D. G. Grier, The Charge of Glass and Silica Surfaces, J. Chem. Phys., 2001, 115, 6716-6721.

27 M. Wang and A. Revil, Electrochemical Charge of Silica Surfaces at High Ionic Strength in Narrow Channels, J. Colloid Interface Sci., 2010, 343, 381-386.

28 G. Manning, Counterion Condensation Theory of Attraction between Like Charges in the Absence of Multivalent Counterions, Eur. Phys. J. E: Soft Matter Biol. Phys., 2011, 34, 1-18.

29 T. A. Gmür, A. Goel and M. A. Brown, Quantifying Specific Ion Effects on the Surface Potential and Charge Density at Silica Nanoparticle-Aqueous Electrolyte Interfaces, J. Phys. Chem. C, 2016, 120, 16617-16625.

30 S. Hocine, R. Hartkamp, B. Siboulet, M. Duvail, B. Coasne, P. Turq and J.-F. Dufrêche, How Ion Condensation Occurs at a Charged Surface: A Molecular Dynamics Investigation of the Stern Layer for Water-Silica Interfaces, J. Phys. Chem. C, 2016, 120, 963-973.

31 X. Wu, W. Lu, W. Ou, M. C. Caumon and J. Dubessy, Temperature and Salinity Effects on the Raman Scattering Cross Section of the Water $\mathrm{OH}$-Stretching Vibration Band in $\mathrm{NaCl}$ Aqueous Solutions from 0 to $300{ }^{\circ} \mathrm{C}$, J. Raman Spectrosc., 2016, 48, 314-322.

32 J. E. Bertie and Z. Lan, Infrared Intensities of Liquids XX: The Intensity of the $\mathrm{OH}$ Stretching Band of Liquid Water Revisited, and the Best Current Values of the Optical Constants of $\mathrm{H}_{2} \mathrm{O}(\mathrm{l})$ at $25{ }^{\circ} \mathrm{C}$ between 15000 and $1 \mathrm{~cm}^{-1}$, Appl. Spectrosc., 1996, 50, 1047-1057. 